\title{
Investigation and Research on User Demand for Electric Vehicles Used in Takeout Food Delivery in China
}

\author{
Yi Feng \\ College of Arts and Design, Beijing Forestry University, Beijing, China
}

Keywords: vehicles used in takeout food delivery, market survey, user demand.

\begin{abstract}
The methods of shadow study, observation and in-depth interviews are used in this research to understand users' demands on electric vehicles in Chinese market through analyzing the behaviors of takeout food deliverymen. With the continuous development of science, technology and the Internet industry, applications have become an integral part of our life. A number of new industries, such as the takeout delivery industry, has come into being. Electric vehicles used today cannot meet the requirements of takeout food delivery. Through survey and interviews with food deliverymen, as well as the collection of literature and comparison of similar products, this article concludes that, Chinese market requires special electric vehicles used in takeout food delivery; suggestions on the improvement of electric vehicles are provided accordingly.
\end{abstract}

\section{Introduction}

\subsection{Current situation of electric vehicles used in takeout food delivery}

With the continuous development of science, technology and the Internet industry, applications have become an integral part of our life. The change brings great convenience for people and drives the development of a series of new industries, such as the takeaway food delivery industry. In the first half of 2016, iResearch issued the Development Report on O2O Takeout Food Industry of China in 2016. The report shows that the whole food and beverage market has reached the volume of hundreds of billions. In 2015, iResearch data showed that the scale of takeout food market exceeded 230 billion, accounting for $7.4 \%$ of the total food and beverage consumption. By 2018, this proportion is expected to reach $14.8 \%$ while the overall size of the takeout market is expected to exceed 660 billion. According to the data released by the largest domestic catering business platform, Meituan, the website has covered more than 200 cities in China, with its maximum trading volume per day exceeded 70 million yuan and the trading volume per month exceeded 1.7 billion yuan. These data and phenomena indicate that; online ordering of takeaway food has formed a huge consumption market. The rise of takeout food industry has led to a large influx of food deliverymen. Electric vehicles are necessary in the process of delivering and distributing takeout food; they are the most important equipments in food delivery.

\subsection{Research background}

The main process of takeout food delivery goes as following. First of all, the customer order food on the platform. Then the platform assigns the delivery task to deliveryman. The deliveryman go to the restaurant to take the food and then deliver it to the customer. In towns and cities, can see delivery men on streets and read reports about them quite often. They cause many traffic accidents when they rush to deliver the food or answer phones while driving. The work of food delivery is not safe. At present, most of the takeout food delivery vehicles in China are refitted by ordinary electric vehicles. Some big platforms post advertisements on the electric vehicles, but fail to change the form of takeaway vehicles. In the market, there is no design for vehicles or related facilities target for serving the takeout food delivery industry. Electric vehicles used in takeaway food delivery are facing many design shortcomings. 


\section{Research Methods}

First of all, our team members investigate takeaway deliverymen. According to the survey results, deliverymen are quite busy. They need to work outside every day and it is difficult for them to live a regular life in the open air environment. The time of work is very concentrated, usually focuses in the two hours around the midday and supper time. The locations of deliverymen are scattered. They need to go to a number of places to take and deliver the food, which brings a lot of hidden troubles. The use of mobile phones in delivery also brings security risks. In the open air environment, weather emergencies are frequent but they cannot hide.

The members of the research group edit a story about the life of a takeout food deliveryman according to observation and imagination. The selected protagonist is a takeaway deliveryman of Meituan. His is 28 years old, and he has worked for one year. He has to face six main problems in working process.

1) There is no place to store his personal stuff.

2) Seats are too hot during summer.

3) Food will be spilled due to road bumps. Customers may complain or even make negative comments.

4) There is no way to answer or make telephone calls when driving.

5) In the case of bad weather, there is no place to hide

6) There is no place to sit and have meals.

Following research is carried out according to these hypothetical questions.

\section{Investigation Implementation and Result Analysis}

\subsection{Investigation implementation}

User survey. During the rush hours of lunch and supper, we do the survey and observation about the deliverymen in dormitory buildings, in order to understand their lives and excavate problems about delivery vehicle. The main purpose of our research is to understand these problems through brainstorming and speculation of problems in the story. The interview is mainly divided into two aspects. First, we observe the work process of takeaway deliverymen, understand problems they encounter and analyze their potential needs. Second, we conduct in-depth interviews with deliverymen and record data, in order to find out the real needs of them.

We interview four deliverymen and learn following facts about them. They are very busy in rush hour, and they usually have lunch after 2 p.m. Different takeout platforms have different processes of order receiving. In Meituan, the mission is assigned to deliverymen; in Eleme, deliverymen have to compete to get the order. Sometimes they need to stop the vehicle and compete the order by the road. It is very inconvenient for them to use mobile phone in the course of driving. They have raincoats provided by the platform companies. But when the weather suddenly changes, there is nowhere to hide. During the interview, many of them express that the battery of electric vehicles consume too fast. They need to charge the vehicle every four hours. There is no place to store the takeaway box, which is inconvenient for them. Another problem is that; customers' meals always spill out if the road is bumpy. Customers may publish bad comments about the deliveryman. In the interview, a deliveryman expresses, now many universities refuse takeout delivery vehicles to enter the campus. They have to leave their cars and walk into the school, which costs much more time.

When observing the working situation of food deliverymen, it is found that the frequent use of mobile phones cause a lot of safety hazards; they may spill the meal which brings complain or bad comments from customers. It is also found that their faces and hands turn red because of long time working outdoors in the cold winter.

Through the results of user investigation, our group discusses and analyzes the preliminary design elements: storage capacity, auxiliary food offering device, portable battery and sensitive brake, in order to solve the inconvenience in bad weather, combine delivery vehicles with mobile phones, store multiple takeaway boxes, and realize the stability of delivery vehicles. 
Analysis of competitive products. This paper compares the delivery vehicles at home and abroad. In order to improve the efficiency of food delivery, the takeaway platform Eleme first remold the delivery vehicle. They choose the double boxes electric vehicles. An electric vehicle can be equipped with two types of takeaway vehicles, type 181 and type 431, and can carry more than 50 orders of takeout food at the same time. Small boxes which can be carried by deliverymen on shoulders also bring great convenience. The Meituan group draws lessons and adopts the two boxes electric vehicles. They also provide a new network for battery replacement. Most of the takeaway delivery vehicles in China have advertisements posted on their appearance. According to the survey, now in our country, most of the delivery vehicles are converted from ordinary electric vehicles through back seat refitting. Deliverymen think that takeaway vehicles provided by the platform are relatively expensive and the battery drains too fast. Foreign delivery vehicles are very different from those in China. In foreign countries, takeout delivery vehicles are provided by merchants, so they are quite different in design. Delivery vehicles drove by intelligent robots are also available. The design makes the delivery process more safety, and provides a more comfortable working environment for deliverymen.

\subsection{Research results analysis}

Through the implementation of survey in the research, it is found that a takeaway deliveryman faces following problems in his work.

A) There is no shelter in rainy days.

B) There is no place to store their personal stuff.

C) During the delivery process, the use of mobile phones increases security risks.

D) Food and drink spills due to road bumps.

E) The storage and usage of delivery box is not reasonable.

According to the investigation and study of takeaway vehicles at home and abroad, it is found that the structure of delivery vehicles of Eleme greatly improves the efficiency of food delivery, while Meituan uses the backup power grid system to solve the problem of battery drain. But these two platforms have too many takeaway orders; exclusive takeaway vehicles can't meet their equipments. Huang Taiji's takeaway vehicles look like walking billboards. The company invests a lot of money to make its brand characteristics. In foreign countries, takeout delivery vehicles are more intelligent because they are provided by restaurants. These vehicles are relatively different; they are more professional and more suitable for the working environment of deliverymen. The appearances of domestic takeaway delivery vehicles are quite similar. Vehicles of different takeaway platforms cannot be distinguished by external appearances.

The research found that, delivery vehicles of different platforms can only be identified from the color and brand identity. In foreign countries, the appearances of takeaway delivery vehicles are relatively different; these vehicles are becoming more intellectualized. Through the results of research and investigation, our group discusses and analyzes the preliminary design elements: storage capacity, auxiliary food offering device, portable battery and sensitive brake, in order to solve the inconvenience in bad weather, combine delivery vehicles with mobile phones, store multiple takeaway boxes, and realize the stability of delivery vehicles.

\section{Suggestions on the Design of Food Delivery Vehicles}

From above investigation and research, it is found that takeaway delivery vehicles in China needs to be improved. The design of takeaway delivery vehicle should start from deliveryman and try to solve their problems at work. We can improve the delivery vehicles in our country from two aspects, so that the takeout food deliverymen in China can have a more comfortable, safe and convenient working environment.

\subsection{Improving the humanized design of food delivery vehicle}

We can add a partition in the delivery box to solve the problem of spilled meal due to uneven roads. A closed food delivery car can be designed and used to prevent deliverymen from suffering in bad weathers, and help them store their personal stuff. Adding mobile phone bracket or more intelligent 
facilities can make the delivery process more safety. Besides, takeaway delivery vehicles in China should also be combined with science and technology fruits, and develop in the direction of intelligence.

\subsection{Improving the design of supporting facilities}

At the present stage, the economic bases of deliverymen in our country are not solid. The large number of delivery vehicles in the whole country requires improved supporting facilities. For example, in order to eliminate insecurity brought by mobile phone using in the course of driving, an accessory that is affiliated with the delivery vehicle can be designed. For the problem of rainy days, shelters can be designed to prevent deliverymen from suffering in bad weathers. For the problem of take-out boxes, supporting facilities to increase the length or height of the back seat of takeaway food delivery vehicles can be provided.

\section{References}

[1] J. van der Geer, J.A.J. Hanraads, R.A. Lupton, the art of writing a scientific article, J. Sci. [1] X. Lin, P. Wells, B. K. Sovacool, Benign mobility? Electric bicycles, sustainable transport consumption behavior and socio-technical transitions in Nanjing, China, J. Transportation Research Part A. 103(2017).

[2] T.Q. Tang, X.F. Luo, J. Zhang, L. Chen. Modeling electric bicycle's lane-changing and retrograde behaviors, J. Physica A: Statistical Mechanics and its Applications. 2017.

[3] S. Liang, S. Wang, X.F. Yang, G.H. Liu, The application of GIS in modern logistics static path planning, J. Standardization of Surveying and Mapping. 29 (2013).

[4] Y. Gu, Y.T. Yang, An economic inquiry into the promotion of electric bicycles in China from the perspective of urban transportation, J. Journal of Lanzhou University (Social Sciences). 40 (2012).

[5] H.X. Pan, Evolution and sustainable development of traffic policies about bicycle in cities of China, J. Urban Planning Forum. 04 (2011).

[6] J. Chen, Z.Q. Xie, Bicycle traffic model for pedestrian-bicycle sharing roads, J. Journal of Jilin University (Engineering and Technology Edition). 39 (2009).

[7] J.C. Xianyu, Z.C. Juan, Choice of transpiration means based on individual differences and preference set, J. Statistics and Decision. 14 (2009).

[8] L.W. Dang, Optimization Research for Just-in-time Material Delivery for Discrete Production System, Shanghai Jiao Tong University, 2013.

[9] F. Xue, Research on delivery optimization of S company based on the economical method, Shanxi University, 2011. 